\section{More on the statistical treatment of individual differences in multidimensional scaling}

\author{
JAMES H. HOWARD, JR. \\ The Catholic University of America \\ Washington, D.C. 20064
}

In a recent note, O'Hare (1977) raised an interesting objection regarding the use of multidimensional scaling procedures for the assessment of individual or group differences. The main thrust of his argument is that "visual inspection of the subject space, obtained for instance from the INDSCAL procedure, or the application of any kind of linear statistics, are liable to give misleading results" (p. 283). His point is that these methods do not consider individual differences in the total proportion of variance accounted for. Two observers or groups of observers occupying the same relative positions in the subject space would tend to diverge as their total proportion of variance accounted for increased. This condition is illustrated in the hypothetical two-dimensional subject space presented in Figures $1 \mathrm{~A}$ and 1B. The solution presented in $1 \mathrm{~B}$ accounts for a larger proportion of the overall variability than the solution presented in 1A (cf. Carroll \& Wish, 1974). Although the relative emphasis placed on the two dimensions is identical in the two solutions, the distance between the two groups is larger in the "good fit" solution (i.e., Figure 1B) than in the "poor fit" solution (i.e., Figure 1A). As an alternative to linear statistics, O'Hare recommends the use of "directional statistics" (Mardia, 1972) which effectively ignore the length of each individual weight vector and, hence, the total proportion of variance accounted for. Some guidance on the proper statistical treatment of individual differences is timely and appropriate; however, a few cautions on the application of directional statistics to the analysis of these data should be noted. In addition, O'Hare's specific criticisms of a paper by Howard and Silverman (1976) are somewhat misleading and deserve further clarification.

First, the application of directional statistics, as any statistical procedure, should not be undertaken without careful consideration of the desired goals of the study. In particular, since the recommended techniques examine only the direction of individual weight vectors in the subject space (i.e., the relative importance of different dimensions) and ignore

The author acknowledges the stimulating discussion of this issue with Donald C. Burgy, John Convey, David J. Getty, David M. Green, Robert P. Gurney, Anthony N. Norcio, and John A. Swets.
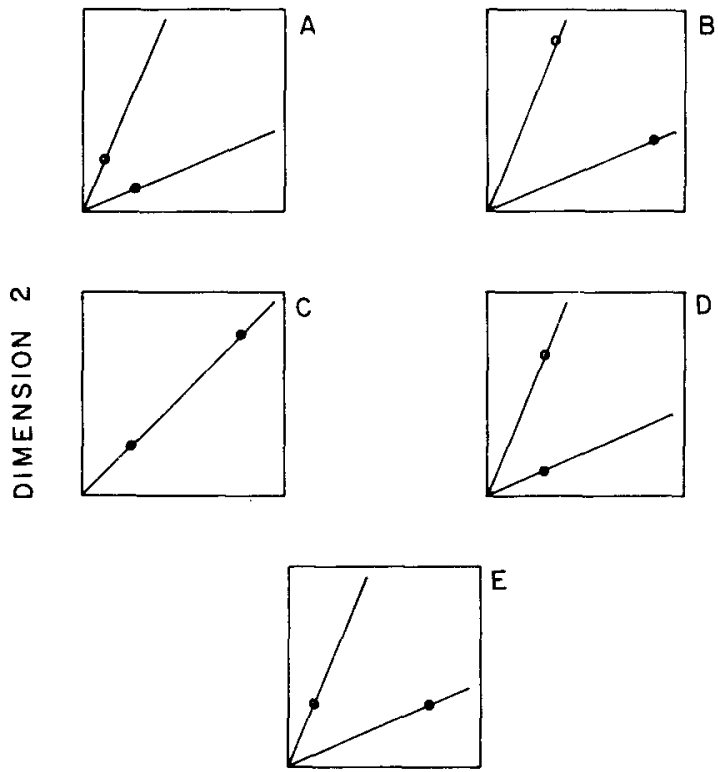

DIMENSION I

Figure 1. Hypothetical two-dimensional subject spaces differing in vector direction (i.e., relative importance of the two dimensions) and length (i.e., approximate proportion of variance accounted for).

vector length (i.e., the approximate proportion of variance accounted for), they are applied with considerable loss of information. While individual differences in the proportion of variance accounted for may be unimportant in some cases, in many-if not most-instances these differences are likely to reflect important differences in perceptual structure. Consider, for example, the hypothetical subject space presented in Figure $1 C$. In this extreme example, the only difference between the two groups is in the total variance accounted for. That this difference would lead to performance differences in a perceptual task involving these stimuli seems obvious. Similarly, the hypothetical data displayed in Figures 1D and 1E would not be distinguished by the exclusive use of the suggested procedures. In this case, the relative directions of the two groups are identical in the two figures, but the perceptual structure is dramatically different.

In short, although the recommended directional techniques are appropriate for some analyses, they do not provide sufficient information for the analysis of individual or group differences in the general case. In most situations where multidimensional scaling techniques are applied to the analysis of perceptual data, it is not sufficient to determine that individuals or groups differ, but rather, it is also of interest to 
establish how they differ. Although it is clearly important to distinguish between direction and length parameters as $O$ 'Hare suggests, further analyses are necessary to provide a complete analysis.

Second, two points should be noted on O'Hare's specific criticism of the Howard and Silverman (1976) study:

(1) Despite the implication of O'Hare's paper to the contrary, the analysis of perceptual differences between musically experienced and musically inexperienced listeners was not the central focus of the paper. As was clearly indicated in the paper, the analysis of musical background was based on a post hoc division of the 34 listeners into two categories according to a rough definition of "musical experience." The primary purpose of this secondary analysis was to provide information of a heuristic, rather than inductive or deductive, nature. It was for this reason that "any supportive statistical analysis is conspicuously absent" (O'Hare, 1977; p. 283).

(2) O'Hare's statistical reanalysis of our group data using directional statistics seems inappropriate given our heuristic conclusion. The point we were making was that while most listeners placed a primary emphasis on the fundamental frequency dimension (i.e., overall, points in the subject space were displaced outward along this dimension), two main clusters of subjects could be identified in the space for Dimensions 1 and 3. Our Figure 7 is replotted in Figure 2 with the two primary clusters identified in a subsequent hierarchical clustering analysis of the intersubject distances (Johnson, 1967; connectedness method). While we admittedly made no attempt to examine vector length and direction separately, the fact that 9 of the 11 musically experienced observers fell into the same category, while the musically inexperienced observers were split between the two categories (11 and 12 in the two clusters, respectively) seemed worth noting for heuristic reasons. Given the tenuous, post hoc definition of musical experience and the substantial overlap in the distributions of musical and nonmusical listeners, it should not be surprising that an analysis of directional differences in the central tendencies of these groups would not be significant, regardless of whether "directional" or linear techniques are

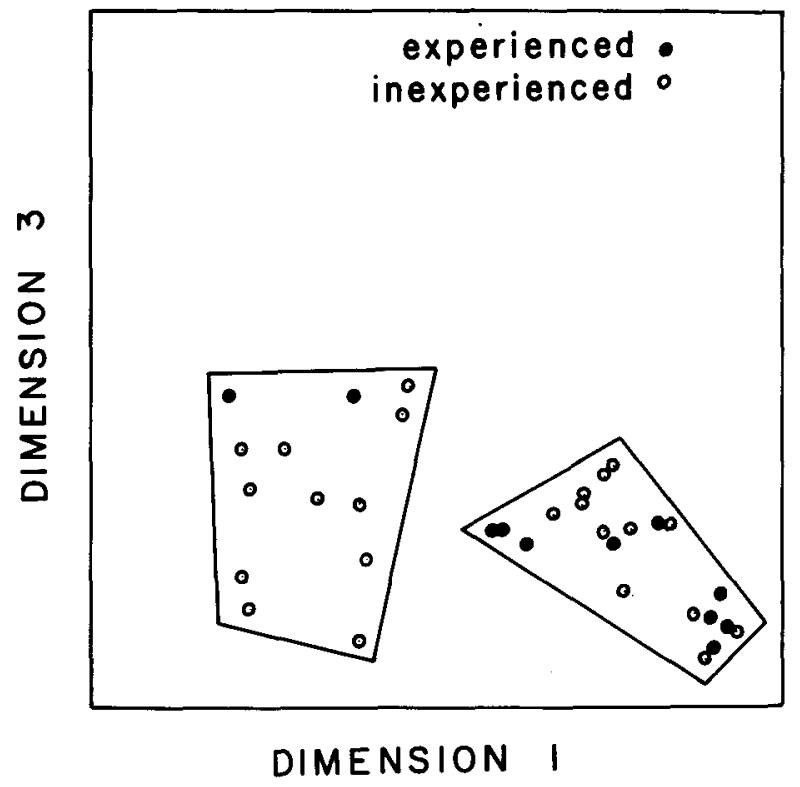

Figure 2. Two-dimensional subject saliency space for musically experienced and musically inexperienced listeners showing the two primary clusters identified in a hierarchical clustering analysis.

applied. That we were trying to clarify and not conceal this fact is precisely the reason why we chose to include both a plot of the subject space (our Figure 7) and the individual listener weights in tabular form (our Table 3).

\section{REFERENCES}

Carroll, J. D., \& WISH, M. Models and methods for three-way multidimensional scaling. In D. H. Krantz, R. C. Atkinson, R. D. Luce, \& P. Suppes (Eds.), Measurement, psychophysics, and neural information processing (Vol. II). San Francisco: Freeman, 1974.

Howard, J. H., \& Silverman, E. B. A multidimensional scaling analysis of 16 complex sounds. Perception \& Psychophysics, 1976, 19, 193-200.

Jornson, S. C. Hierarchical clustering schemes. Psychometrika, $1967,32,241-254$.

Mardia, K. V. Statistics of directional data. London: Academic Press, 1972.

O'HARE, D. P. A note on the statistical treatment of individual differences in multidimensional scaling. Perception \& Psychophysics, 1977, 21, 283-284.

(Received for publication May 6, 1977; accepted May 10, 1977.) 